\title{
Targeted Therapy in the Management of Elderly Patients with Pancreatic Metastases from Renal Cell Carcinoma*
}

\author{
Keith Chiu ${ }^{1 \#}$, Abdul Razack ${ }^{1}$, Anthony Maraveyas ${ }^{2}$ \\ ${ }^{1}$ Department of Radiology, Hull and East Yorkshire NHS Trust, Hull, UK; ${ }^{2}$ The Queen's Centre for Oncology and Haematology, \\ Hull and York Medical School, Castle Hill Hospital, Hull, UK. \\ Email: "keith.chiu@hey.nhs.uk
}

Received July $26^{\text {th }}, 2013$; revised August $24^{\text {th }}, 2013$; accepted September $1^{\text {st }}, 2013$

Copyright (c) 2013 Keith Chiu et al. This is an open access article distributed under the Creative Commons Attribution License, which permits unrestricted use, distribution, and reproduction in any medium, provided the original work is properly cited.

\begin{abstract}
Background: The pancreas is an uncommon but recognizable site for metastases from renal cell carcinoma (RCC). Isolated pancreatic RCC metastases are still rarer and often present years after initial nephrectomy. Surgical resection has been the treatment of choice because of superior patient survival compared with traditional immunotherapy. In recent years, the advent of targeted therapy has transformed the outcomes of patients with metastatic RCC although little evidence is available on its effectiveness on this subset of patients. We report our experience of 6 patients with pancreatic RCC metastases. Patients and Methods: Between 2007 and 2012, 6 patients (2 men, 4 women; median age 78 years) were diagnosed to have pancreatic RCC metastases at our institute. The clinical features, treatment and outcomes were examined. Results: All 6 patients had a primary RCC of clear cell type. The median interval between initial curative nephrectomy and re-presentation with pancreatic metastases was 12.5 years. Four patients were asymptomatic at the time of diagnosis, one presented with obstructive jaundice and another with acute gastrointestinal bleed. Four patients had extra-pancreatic disease. All were deemed unsuitable or unfit for surgical metastasectomy. Five patients had a Memorial Sloan-Kettering Cancer Center (MSKCC) score of 1 (moderate risk) and the other patient had a score of 0 (good risk). Two patients were commenced on Sunitinib, one received Pazopanib and one received Temsirolimus. Two patients did not undergo further treatment. Of the 4 patients who underwent targeted therapy, the median follow up was 33 months with a median progression free survival of 16 months. One achieved complete response but recurred soon after treatment was stopped. Targetted therapy was recommenced and the disease remained stable. A second patient had long period of stable disease before disease progression. A third achieved partial response since started on targeted therapy and a fourth had disease progression despite treatment. Of the four patients who underwent systemic therapy, three are still alive at the time of this report. Conclusion: Pancreatic metastasis from RCC is a unique subgroup of disease which runs an indolent course, and a higher incidence in an elderly population. Our results demonstrate that targeted therapy can be efficacious in some patients where surgical resection is not suitable or possible.
\end{abstract}

Keywords: Isolated Pancreatic Metastases; Renal Cell Carcinoma; Targeted Therapy

\section{Introduction}

Pancreatic metastases are rare and renal cell carcinoma (RCC) is one of the few known tumours that metastasize to this site [1]. They can either present as part of disseminated disease or as isolated metastases. Historically, disease response and survival rates in patients on immunotherapy-type systemic therapy have been disappointing [2-8], and surgical metastasectomy has been advocated in

*Funding/Grant: N/A.

Author Contribution: Chiu K, Razack A and Maraveyas A contributed equally to this work. All three researched, wrote and edited the manuscript.

${ }^{\#}$ Corresponding author. patients with resectable disease as they have shown superior outcomes [9-12].

The introduction of targeted therapy has transformed the outcomes of patients with metastatic RCC (mRCC). Several clinical trials have shown that multi-targeted receptor tyrosine kinase (RTK) inhibitor such as sunitinib, pazopanib or monoclonal antibody avastin based biotherapies have improved both the progression free (PFS) and overall (OS) survival of these patients. For example, Motzer's multi-centre randomized phase III trial has shown that sunitinib doubled the median progression free survival (PFS) when compared with immunotherapy such as interferon $[13,14]$. Similarly, Sternberg and colleagues 
have found that pazopanib doubled the median PFS when compared with placebo but even more impressively, in treatment naïve patients, the median PFS quadrupled [15]. More recently, the COMPARZ trial has shown that both pazopanib and sunitinib are equally effective [16]. These agents have now become the current standard of care for first line treatment of mRCC $[17,18]$.

Pancreatic metastases from RCC tend to be principally metachronous events. Although these patients may suffer from particular complications (such as biliary tree obstruction) due to its complex relation with its surrounding structures, current literature seems to suggest that they tend to have good prognostic indices and have a "slow-burning" course [19]. Real world data seem to suggest that RTK inhibitors can benefit patients across the spectrum of performance status and age although little is known about particular cohorts of patients with rare metastatic sites like the pancreas [20].

\section{Materials and Methods}

Between 2007 and 2012, 6 patients (2 men, 4 women; median age 78 years; range 59 - 80 years) were treated by the Department of Oncology in Hull and East Yorkshire NHS Trust. Primary tumour histology, interval between primary treatment and re-presentation, presenting complaints and symptoms, diagnostic imaging and clinical outcomes including response rate and treatment toxicity were evaluated. Statistical analysis was performed with Graphpad Prism 5 statistical software package (GraphPad. Software, La Jolla, CA).

\section{Results}

The demographics and clinical features of the 6 patients are summarized in Table 1. The median interval between initial presentation of primary tumour and diagnosis of pancreatic metastases was 12.5 years (range: 2 - 22 years). Four patients were asymptomatic at the time of presentation; three were discovered during routine follow-up CT scans and one was an incidental finding on a CT for suspected lung carcinoma. Two patients were symptomatic at presentation, one with obstructive jaundice and the other acute upper gastrointestinal haemorrhage. Histological sample was obtained in all 6 patients with 5 underwent endoscopic ultrasonography fine needle aspiration and one percutaneous ultrasound guided pancreatic biopsy. All 6 patients had clear cell renal cell carcinoma in their initial tumours and pancreatic metastases (Figure 1).

The patient who presented with obstructive jaundice had multiple metastases in the pancreas but no extrapancreatic disease. He underwent percutaneous cholangiography and metallic biliary stent insertion to relieve his jaundice and was commenced on pazopanib (Figure 2). The other patient who presented with acute upper gastrointestinal haemorrhage also had diffuse pancreatic metastases and also single renal metastases in her contralateral kidney. She underwent tumour embolization to control the bleeding and was treated with sunitinib (Figure 3).

Of the four asymptomatic patients, one had diffuse pancreatic metastases and a single liver metastasis. Due to his extensive cardiac history, he was commenced on

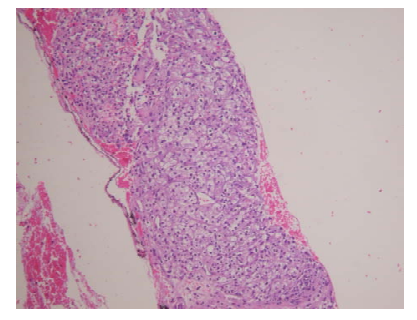

(a)

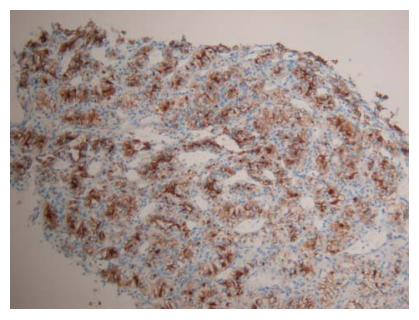

(b)
Figure 1. (a) Histology from endoscopic ultrasound biopsy demonstrating classical appearance of clear cell RCC metastasis. (b) High expression of membranous claudin-1 protein in clear cell RCC.

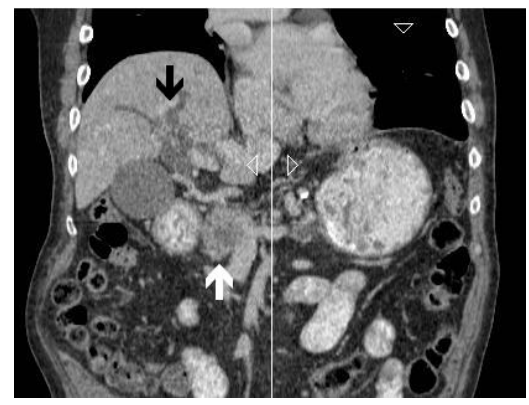

Figure 2. Patient presented with obstructive jaundice. Coronal CT image demonstrated intrahepatic duct dilatation (black arrow) secondary to metastasis at the head of pancreas (white arrow). Biopsy from endoscopic ultrasound confirmed clear cell RCC metastasis.

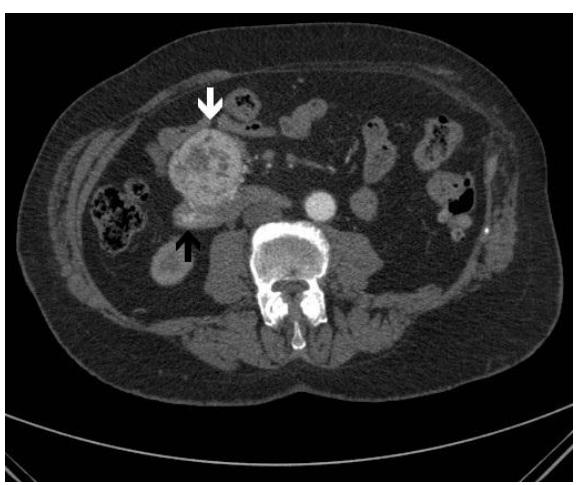

Figure 3. Patient presented with acute upper GI haemorrhage. Axial CT image showed the lesion has eroded through into the duodenum (white arrow). Active contrast extravasion indicating active haemorrhage (black arrow) was seen in the second and third part of the duodenum. The patient underwent tumour embolization for haemostasis. 


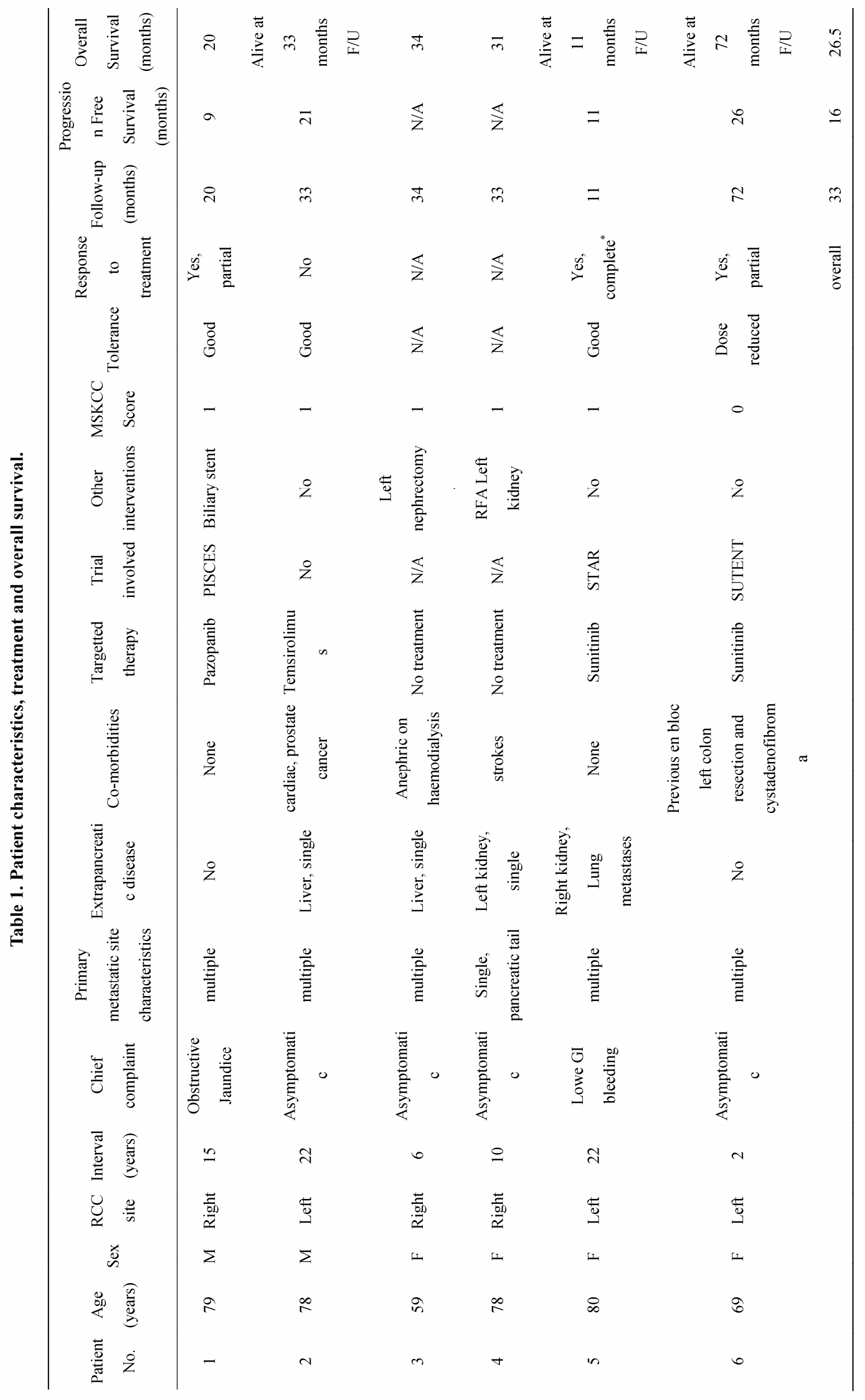


Temsirolimus. Another patient also had multiple pancreatic metastases but no extra-pancreatic disease. She was commenced on sunitinib. One of the remaining two patients had a solitary pancreatic metastasis but also a synchronous metastasis in the contralateral kidney. This patient was not suitable for systemic therapy due to her neurological comorbidities (several episodes of strokes) but underwent two radiofrequency ablation of the renal metastasis in her contralateral kidney. The other patient that did not receive any systemic therapy had multiple pancreatic metastases and also a liver metastasis. She was anephric due to nephrectomies for RCC on previous occasions and was on permanent haemodialysis.

The median follow-up of the cohort was 33 months (range 11 - 72 months) after diagnosis of pancreatic RCC metastases. Of the four patients who received systemic therapy, the median follow up was 26 months (range 11 72 months). They underwent regular CT scans to assess treatment response. Of the two patients who did not receive any systemic therapy, one continued to have regular follow-up CT scans for assessment of response from RFA. One patient had no further imaging and was only followed-up clinically and treated palliatively. The patient who was given pazopanib had partial response with a PFS of 9 months. The patient developed stent related biliary sepsis 20 months after initial diagnosis and died from biliary sepsis. The patient who received temsirolimus had a PFS of 21 months before disease progression but is alive at the time of this report (33 months follow-up). Of the two patients who received sunitinib, one achieved complete response after 24 months and sunitinib was stopped. Twelve months after sunitinib was stopped, the patient had recurrence and she was restarted on sunitinib, achieving a partial response and her disease remained stable. The other patient who received sunitinib had stable disease and remained so on follow up after 11 months (Figure 4).

Overall, the median PFS for patients on systemic therapy was 16 months (range: 9 - 26 months) and the median overall survival (OS) was 33 months (range 11 - 72 months). Only one patient who was on systemic therapy died in our series as described above. The two patients who did not receive any systemic treatment passed away 31 and 34 months after initial diagnosis respectively.

\section{Discussion}

Renal cell carcinoma (RCC) is relatively uncommon, representing $2 \%$ of all adult malignancies [21]. Due to the lack of any early warning signs, they often present late with advanced local or metastatic disease. At diagnosis, around $20 \%-30 \%$ of patients have synchronous metastases with a further $40 \%$ initially having localized disease will develop metachronous disease $[1,22,23]$. The pancreas is an unusual site for metastases, accounting for
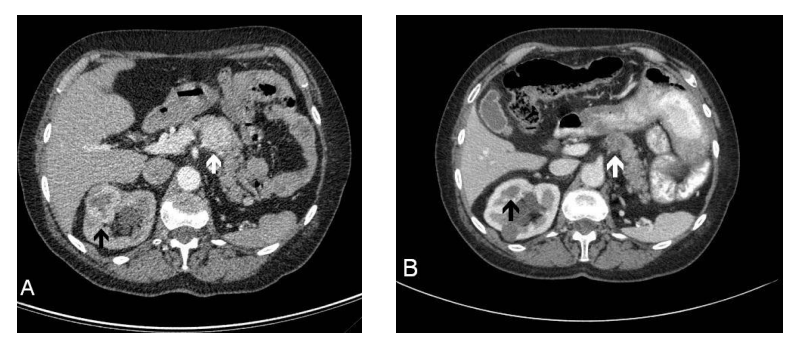

Figure 4. Partial response to sunitinib. On the selected pretreatment axial CT image, a large pancreatic metastasis (white arrow) and a contralateral metastasis in the right kidney (white arrow) were seen. Six months after commencing on sunitinib, both lesions have decreased in size.

only $2 \%-4 \%$ of all pancreatic lesions with colon, lung, breast, malignant melanoma along with RCC being the most common primary tumours $[10,24,25]$.

Although pancreatic metastases are often considered as end stage events, there is a small but distinct group of patients that present with isolated pancreatic RCC metastases. The majority of them are from clear cell type [26-28], and they often present years after initial treatment $[9,10,12]$.

The mechanism with which metastatic RCC spreads to the pancreas is poorly understood. Sellner and colleagues have analyzed a large cohort of pancreatic metastases from RCC and have concluded that haematological spread is the most likely mechanism due to diffuse distribution of pancreatic metastases irrespective of the site of RCC and absence of concurrent metastases to other organs [9].

Surgical resection has been advocated by many studies as the treatment of choice [2-4,9,10,12]. Many case series have demonstrated that resection of metastases in other organs such as liver, lung and brain have improved survival and quality of life [29,30]. In a recent systematic review, Tanis have shown that this is also the case in pancreas. They reviewed 311 patients who underwent pancreatic metastasectomy for mRCC and compared them with 73 patients who were managed medically and have shown that the overall 2 and 5 year survival were significantly higher for the surgical group $(80.6 \%$ and $72.6 \%$ compared to $41 \%$ and $14 \%$ respectively) [12].

The main reasons cited for non-operative management were local irresectability, poor performance status and the presence of extra-pancreatic disease [12]. However, most patients presented with isolated pancreatic metastases usually possess one or more of these factors. The median age for pancreatic metastases from RCC occurs in the $7^{\text {th }}$ decade of life and between $20 \%-45 \%$ of these metastases are multifocal [31]. Our cohort reflects the "real-life" situation where the patients are elderly, and not suitable for surgical intervention due to other comorbidities. The median age at presentation in our cohort was 78 years. In 5 out of our 6 patients, there were dif- 
fuse pancreatic metastases. Three patients had multiple comorbidities precluding them from surgery (one had cardiac disease, one was anephric on haemodialysis after both kidneys were removed due to RCC and another had debilitating neurological co-morbidities as a result of previous episodes of strokes). Four patients had extrapancreatic disease involving the liver, contralateral kidney and lung metastases.

For patients who were not suitable for surgery, they were traditionally treated by immunotherapy such as interleukin-2 or IFNa since RCC is not responsive to traditional chemotherapy. However, the response rate with these agents were often poor (as low as 5\% - 20\%) with the median survival of these patients of usually 12 months and a 5 year survival rate of around $10 \%$ [3,5-8]. In recent years, the emergence of targeted therapy has transformed the management and outcomes of patients with mRCC [14,15,32-34]. The three systemic therapies that were used in our patients have all shown encouraging results. In a randomized phase III trial with 750 patients, Motzer and colleagues had showed the median PFS in patients receiving sunitinib, an oral multi-targeted recaptor tyrosine kinase (RTK) inhibitor, have more than doubled that of IFNa (11 vs 5 months). The OS had also increased by nearly 5 months (26.4 vs 21.8 months) [14]. Similarly, Sternberg and colleagues had demonstrated that pazopanib, another RTK inhibitor, had significantly prolonged the median PFS compared with placebo in treatment naïve patients (9.2 months vs 2.8 months) and achieved a tenfold increase in response rate (30\% vs. $3 \%)$ [15]. As for temsirolimus, a mTOR inhibitor, it was shown to double the median PFS (3.8 vs 1.9 months) months and increased median OS (10.9 vs 7.3 months) when compared to IFNa in a phase III trial [32].

Some of the drawbacks in this study are the small number of patients and the heterogeneous treatment they received with different targeted therapies used. However, isolated pancreatic RCC metastases are rare and although our cohort is small, our results suggest a significant survival benefit when compared to survival of $41 \%$ at 2 years and $14 \%$ at 5 years for the non-surgically treated group in Tanis' systematic review [12], particularly if one factors in the median age and "real world" characteristics of our cohort. With a median PFS of 15 months and median OS of 31 months our data is generally comparable to results from other clinical trials [13-15]. Of the 4 patients who underwent systemic therapy, 3 are still alive at the time of this study with a median follow-up of 26 months (range 9 - 70 months). The two patients who did not receive any further treatment had survived 31 and 34 months after diagnosis underscoring the recognized indolence of the metastatic disease in this group, running a much less aggressive course and having particularly long median overall survival [31].

\section{Conclusion}

Our results in this rare group of patients demonstrate that targeted treatments can be used safely in elderly patients who are usually unfit for surgery or their disease is unsuitable for pancreatic resection and can improve outcomes even in these better prognosis metastatic patients.

\section{REFERENCES}

[1] S. L. Zweizig, "Cancer of the Kidney,” Clinical Obstetrics and Gynecology, Vol. 45, No. 3, 2002, pp. 884-891. http://dx.doi.org/10.1097/00003081-200209000-00035

[2] S. Pyrhonen, E. Salminen, M. Ruutu, T. Lehtonen, M. Nurmi, T. Tammela, et al., "Prospective Randomized Trial of Interferon Alfa-2a Plus Vinblastine versus Vinblastine Alone in Patients with Advanced Renal Cell Cancer,” Journal of Clinical Oncology, Vol. 17, No. 9, 1999, pp. 2859-2867.

[3] R. J. Motzer, B. A. Murphy, J. Bacik, L. H. Schwartz, D. M. Nanus, T. Mariani, et al., "Phase III Trial of Interferon Alfa-2a with or without 13-Cis-Retinoic Acid for Patients with Advanced Renal Cell Carcinoma,” Journal of Clinical Oncology, Vol. 18, No. 16, 2000, pp. 2972-2980.

[4] W. M. Stadler, T. Kuzel, M. Dumas and N. J. Vogelzang, "Multicenter Phase II Trial of Interleukin-2, InterferonAlpha, and 13-Cis-Retinoic Acid in Patients with Metastatic Renal-Cell Carcinoma,” Journal of Clinical Oncology, Vol. 16, No. 5, 1998, pp. 1820-1825.

[5] R. I. Fisher, S. A. Rosenberg and G. Fyfe, "Long-Term Survival Update for High-Dose Recombinant Interleukin-2 in Patients with Renal Cell Carcinoma,” The Cancer Journal From Scientific American, Vol. 6, Suppl. 1, 2000, pp. S55-S57.

[6] D. F. McDermott, M. M. Regan, J. I. Clark, L. E. Flaherty, G. R. Weiss, T. F. Logan, et al., "Randomized Phase III Trial of High-Dose Interleukin-2 versus Subcutaneous Interleukin-2 and Interferon in Patients with Metastatic Renal Cell Carcinoma,” Journal of Clinical Oncology, Vol. 23, No. 1, 2005, pp. 133-141. http://dx.doi.org/10.1200/JCO.2005.03.206

[7] S. Negrier, B. Escudier, C. Lasset, J. Y. Douillard, J. Savary, C. Chevreau, et al., "Recombinant Human Interleukin-2, Recombinant Human Interferon Alfa-2a, or Both in Metastatic Renal-Cell Carcinoma,” The New England Journal of Medicine, Vol. 338, No. 18, 1998, pp. 1272-1278.

http://dx.doi.org/10.1056/NEJM199804303381805

[8] J. C. Yang, R. M. Sherry, S. M. Steinberg, S. L. Topalian, D. J. Schwartzentruber, P. Hwu, et al., "Randomized Study of High-Dose and Low-Dose Interleukin-2 in Patients with Metastatic Renal Cancer,” Journal of Clinical Oncology, Vol. 21, No. 16, 2003, pp. 3127-3132. http://dx.doi.org/10.1200/JCO.2003.02.122

[9] F. Sellner, N. Tykalsky, M. de Santis, J. Pont and M. Klimpfinger "Solitary and Multiple Isolated Metastases of Clear Cell Renal Carcinoma to the Pancreas: An Indication for Pancreatic Surgery," Annals of Surgical Oncology, Vol. 13, No. 1, 2006, pp. 75-85. 
http://dx.doi.org/10.1245/ASO.2006.03.064

[10] O. Strobel, T. Hackert, W. Hartwig, F. Bergmann, U. Hinz, M. N. Wente, et al., "Survival Data Justifies Resection for Pancreatic Metastases,” Annals of Surgical Oncology, Vol. 16, No. 12, 2009, pp. 3340-3349. http://dx.doi.org/10.1245/s10434-009-0682-3

[11] A. D. Sweeney, M. F. Wu, S. G. Hilsenbeck, F. C. Brunicardi, W. E. Fisher, "Value of Pancreatic Resection for Cancer Metastatic to the Pancreas," Journal of Surgical Research, Vol. 156, No. 2, 2009, pp. 189-198. http://dx.doi.org/10.1016/j.jss.2009.01.017

[12] P. J. Tanis, N. A. van der Gaag, O. R. Busch, T. M. van Gulik and D. J. Gouma, "Systematic Review of Pancreatic Surgery for Metastatic Renal Cell Carcinoma,” British Journal of Surgery, Vol. 96, No. 6, 2009, pp. 579-592. http://dx.doi.org/10.1002/bjs.6606

[13] R. J. Motzer, T. E. Hutson, P. Tomczak, M. D. Michaelson, R. M. Bukowski, O. Rixe, et al., "Sunitinib versus Interferon Alfa in Metastatic Renal-Cell Carcinoma,” The New England Journal of Medicine, Vol. 356, No. 2, 2007, pp. 115-124. http://dx.doi.org/10.1056/NEJMoa065044

[14] R. J. Motzer, T. E. Hutson, P. Tomczak, M. D. Michaelson, R. M. Bukowski, S. Oudard, et al., "Overall Survival and Updated Results for Sunitinib Compared with Interferon Alfa in Patients with Metastatic Renal Cell Carcinoma," Journal of Clinical Oncology, Vol. 27, No. 22, 2009, pp. 3584-3590. http://dx.doi.org/10.1200/JCO.2008.20.1293

[15] C. N. Sternberg, I. D. Davis, J. Mardiak, C. Szczylik, E. Lee, J. Wagstaff, et al., "Pazopanib in Locally Advanced or Metastatic Renal Cell Carcinoma: Results of a Randomized Phase III Trial,” Journal of Clinical Oncology, Vol. 28, No. 6, 2010, pp. 1061-1068. http://dx.doi.org/10.1200/JCO.2009.23.9764

[16] A. Ceschia, “2012 ESMO Congress,” The Lancet Oncology, Vol. 13, No. 11, 2012, p. 1081.

[17] B. Escudier and V. Kataja, "Renal Cell Carcinoma: ESMO Clinical Practice Guidelines for Diagnosis, Treatment and Follow-Up,” Annals of Oncology, Vol. 21, Suppl. 5, 2010, pp. v137-v139.

http://dx.doi.org/10.1093/annonc/mdq206

[18] National Institute for Health and Clinical Excellence, "Sunitinib for the First-Line Treatment of Advanced and/or Metastatic Renal Cell Carcinoma (TA169),” National Institute for Health and Clinical Excellence, London, 2009.

[19] J. P. Faure, J. J. Tuech, J. P. Richer, P. Pessaux, J. P. Arnaud and M. Carretier "Pancreatic Metastasis of Renal Cell Carcinoma: Presentation, Treatment and Survival," Journal of Urology, Vol. 165, No. 1, 2001, pp. 20-22. http://dx.doi.org/10.1097/00005392-200101000-00005

[20] M. E. Gore, C. Szczylik, C. Porta, S. Bracarda, G. A. Bjarnason, S. Oudard, et al., "Safety and Efficacy of Sunitinib for Metastatic Renal-Cell Carcinoma: An Expanded-Access Trial,” The Lancet Oncology, Vol. 10, No. 8, 2009, pp. 757-763. http://dx.doi.org/10.1016/S1470-2045(09)70162-7

[21] D. M. Parkin, F. Bray, J. Ferlay and P. Pisani, "Global Cancer Statistics, 2002,” CA: A Cancer Journal for Clini- cians, Vol. 55, No. 2, 2005, pp. 74-108. http://dx.doi.org/10.3322/canjclin.55.2.74

[22] R. J. Motzer, N. H. Bander and D. M. Nanus, "Renal-Cell Carcinoma,” The New England Journal of Medicine, Vol. 335, No. 12, 1996, pp. 865-875. http://dx.doi.org/10.1056/NEJM199609193351207

[23] A. Antonelli, A. Cozzoli, D. Zani, T. Zanotelli, M. Nicolai, S. C. Cunico, et al., "The Follow-Up Management of Non-Metastatic Renal Cell Carcinoma: Definition of a Surveillance Protocol,” BJU International, Vol. 99, No. 2, 2007, pp. 296-300.

http://dx.doi.org/10.1111/j.1464-410X.2006.06616.x

[24] C. F. Roland and J. A. van Heerden, "Nonpancreatic Primary Tumors with Metastasis to the Pancreas," Surgery Gynecology \& Obstetrics, Vol. 168, No. 4, 1989, pp. 345347.

[25] K. Z'graggen, C. C. Fernandez-del, D. W. Rattner, H. Sigala and A. L. Warshaw, "Metastases to the Pancreas and Their Surgical Extirpation,” Archives of Surgery, Vol. 133, No. 4, 1998, pp. 413-417. http://dx.doi.org/10.1001/archsurg.133.4.413

[26] A. Kassabian, J. Stein, N. Jabbour, K. Parsa, D. Skinner, D. Parekh, et al. "Renal Cell Carcinoma Metastatic to the Pancreas: A Single-Institution Series and Review of the Literature,” Urology, Vol. 56, No. 2, 2000, pp. 211-215. http://dx.doi.org/10.1016/S0090-4295(00)00639-7

[27] E. G. Robbins, D. Franceschi and J. S. Barkin, "Solitary Metastatic Tumors to the Pancreas: A Case Report and Review of the Literature," The American Journal of Gastroenterology, Vol. 91, No. 11, 1996, pp. 2414-2417.

[28] L. E. Harrison, N. Merchant, A. M. Cohen and M. F. Brennan, "Pancreaticoduodenectomy for Nonperiampullary Primary Tumors,” The American Journal of Surgery, Vol. 174, No. 4, 1997, pp. 393-395. http://dx.doi.org/10.1016/S0002-9610(97)00121-9

[29] R. Kanzaki, M. Higashiyama, A. Fujiwara, T. Tokunaga, J. Maeda, J. Okami, et al., "Long-Term Results of Surgical Resection for Pulmonary Metastasis from Renal Cell Carcinoma: A 25-Year Single-Institution Experience,” European Journal Cardio-Thoracic Surgery, Vol. 39, No. 2, 2011, pp. 167-172. http://dx.doi.org/10.1016/j.ejcts.2010.05.021

[30] M. D. Staehler, J. Kruse, N. Haseke, T. Stadler, A. Roosen, A. Karl, et al., "Liver Resection for Metastatic Disease Prolongs Survival in Renal Cell Carcinoma: 12-Year Results from a Retrospective Comparative Analysis," World Journal of Urology, Vol. 28, No. 4, 2010, pp. 543547. http://dx.doi.org/10.1007/s00345-010-0560-4

[31] R. Ballarin, M. Spaggiari, N. Cautero, N. de Ruvo, R. Montalti, C. Longo, et al., "Pancreatic Metastases from Renal Cell Carcinoma: The State of the Art," World Journal of Gastroenterology, Vol. 17, No. 43, 2011, pp. 47474756. http://dx.doi.org/10.3748/wjg.v17.i43.4747

[32] G. Hudes, M. Carducci, P. Tomczak, J. Dutcher, R. Figlin, A. Kapoor, et al., "Temsirolimus, Interferon Alfa, or Both for Advanced Renal-Cell Carcinoma,” The New England Journal of Medicine, 2007, Vol. 356, No. 22, pp. 22712281. http://dx.doi.org/10.1056/NEJMoa066838

[33] M.E. Gore and J.M. Larkin, “Challenges and Opportuni- 
ties for Converting Renal Cell Carcinoma into a Chronic Disease with Targeted Therapies,” Br J Cancer, Vol. 104, No. 3, 2011, pp. 399-406.

http://dx.doi.org/10.1038/sj.bjc.6606084

[34] B. Escudier, T. Eisen, W.M. Stadler, C. Szczylik, S. Oudard, M. Staehler, et al., "Sorafenib for Treatment of Re- nal Cell Carcinoma: Final Efficacy and Safety Results of the Phase III Treatment Approaches in Renal Cancer Global Evaluation Trial,” Journal of Clinical Oncology, Vol. 27, No. 20, 2009, pp. 3312-3318.

http://dx.doi.org/10.1200/JCO.2008.19.5511 